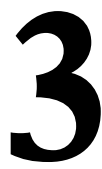

\title{
LA FORMACIÓN INICIAL DEL PROFESORADO DE EDUCACIÓN SECUNDARIA EN ESPAÑA: PERFIL Y MOTIVACIONES DEL FUTURO DOCENTE
}

\author{
(INITIAL TEACHER TRAINING FOR SECONDARY EDUCATION IN SPAIN: \\ PROFILE AND MOTIVATIONS OF THE FUTURE TEACHING STAFF)
}

Guzmán Antonio Muñoz-Fernández

Pablo Rodríguez-Gutiérrez

Universidad de Córdoba

Mercedes Luque-Vílchez

Universidad de Burgos

DOI: $10.5944 / e d u c X X 1.20007$

Cómo referenciar este artículo/How to reference this article:

Muñoz-Fernández, G.A.; Rodríguez-Gutiérrez, P. y Luque-Vílchez, M. (2019). La formación inicial del profesorado de educación secundaria en España: perfil y motivaciones del futuro docente. Educación XX1, 22(1), 71-92, doi: 10.5944/educXX1.20007

Muñoz-Fernández, G.A.; Rodríguez-Gutiérrez, P. \& Luque-Vílchez, M. (2019). La formación inicial del profesorado de educación secundaria en españa: perfil y motivaciones del futuro docente. [Initial teacher training for secondary education in Spain: profile and motivations of the future teaching staff]. Educación XX1, 22(1), 71-92, doi: 10.5944/educXX1.20007

\section{RESUMEN}

La formación inicial del profesorado es un elemento clave para la mejora de la calidad educativa. En este contexto, la necesidad de mejorar las competencias y destrezas del nuevo profesorado, y en concreto, las motivaciones que llevan al futuro docente a la elección del Máster de Formación en Profesorado de Secundaria gozan de una especial relevancia. La importancia de conocer dichas motivaciones reside en su potencial como predictor del desempeño futuro en la docencia. De manera incipiente, la literatura señala la necesidad de estudiar el perfil motivacional en el desarrollo profesional de los docentes. Sin embargo, consideramos que hasta el momento ha sido escasa la atención prestada. En este sentido, el presente trabajo tiene como objetivo profundizar en el análisis del perfil motivacional del alumnado del máster de formación del profesorado. Para ello, se ha realizado una encuesta a 512 alumnas y alumnos de diferentes 
especialidades, en tres universidades españolas (Burgos, Córdoba y Jaén), que respondieron a un cuestionario sobre motivaciones y vocación hacia la docencia. Se ha aplicado un análisis factorial para simplificar y conocer sus principales motivaciones y un análisis clúster para clasificar al alumnado según sus factores motivacionales principales. Los datos revelan que existe relación negativa entre la motivación intrínseca hacia la docencia y la edad del encuestado. También se ha encontrado que el alumnado de áreas de conocimiento relacionadas con las Artes y las Humanidades presenta un mayor nivel de motivación docente intrínseca; y, por otra parte, el hecho de tener un posgrado anterior se relaciona con una menor vocación. Comprender las motivaciones para ser docente, puede tener interés tanto para las autoridades educativas encargadas de la selección del alumnado como para aquellas que tengan la potestad en la planificación de la formación del profesorado, desde el punto de vista del diseño curricular.

\section{PALABRAS CLAVE}

Enseñanza secundaria; formación inicial; motivación; perfil del profesorado.

\section{ABSTRACT}

Initial teacher training is a key element for improving educational quality. The need to improve the skills and abilities of the new teaching staff, and in particular, the motivations that lead the future teacher to choose the masters degree in secondary school teachers are particularly relevant. The importance of knowing these motivations lies in their potential as a predictor of future performance in teaching. In an incipient way, the literature highlights the need to study the motivational profile in the professional development of teachers. However, we consider that the attention given has been scarce so far. In this sense, the objective of this work is to delve further into the motivational analysis of the students of the teacher training masters. To that end, a survey of 512 students of various specialties was carried out in three Spanish universities (Burgos, Córdoba and Jaén), who answered a questionnaire about motivations and vocation for teaching. A factorial analysis was carried out in order to simplify and to learn their main motivations. In addition, a cluster analysis classifies the students according to their main motivation. The data reveals the existence of a negative relationship between both the intrinsic motivation for teaching and the age of the respondent. Moreover, it has also been found that the students of areas of knowledge related to Arts and Humanities show a higher level of intrinsic teacher motivation. On the other hand, the fact of having an earlier postgraduate degree is related to a lower vocation. Thus, understanding the motivations to be a teacher may be of interest to both the educational authorities in charge of the selection of students and those who have the power to plan teacher training from the point of view of curricular design.

\section{KEYWORDS}

Secondary education; initial training; motivation for studies; teaching profile. 


\section{INTRODUCCIÓN}

Cuando se habla de la calidad del sistema educativo, existe un amplio consenso acerca de la importancia que subyace en la preparación del profesorado para que este pueda lograr resultados académicos exitosos en el alumnado (Boyd, Grossman, Lankford, Loeb y Wyckoff, 2009; Harris y Sass, 2011; Musset, 2010). La motivación del profesorado es considerada, junto con otras habilidades, un elemento básico para llegar a ser un buen profesor (Caballero y Huertas, 2016; Comisión Europea, 2013; Ministerio de Educación, Cultura y Deporte, 2014).

La motivación del docente debe compaginarse con circunstancias de muy diversa índole (profesionales y no profesionales) que suponen un problema a la vez que un reto (Lorenzo-Vicente, Muñoz-Galiano y Beas-Miranda, 2015), y que la configuran como una profesión compleja y exigente al mismo tiempo (Ministerio de Educación, Cultura y Deporte, 2014; OCDE, 2005). Por una parte, aparecen nuevas funciones como las TICs y su didáctica, la gestión de los centros educativos, la atención a las necesidades educativas especiales o a la diversidad cultural; y por la otra, retos relacionados con el alumnado, como son la necesidad de resolver problemas de comportamiento y disciplina, y los elevados niveles de fracaso y abandono escolar (Comisión Europea, 2013; León, Domínguez, Núñez, Pérez y Martín-Albo, 2011).

Derivado de lo anterior, puede existir dificultad en disponer de un profesorado «competente» que esté a la altura de los nuevos retos presentes en el sistema educativo (Esteve, 2006). Para hacer frente a estos retos, resultaría necesario analizar el desarrollo (conformación) inicial de la identidad profesional del docente (Bolívar y Moreno, 2006; Hobson, Ashby, Malderez y Tomlinson, 2009).

Sin embargo, hasta el momento pocos estudios han identificado la existencia de diferentes motivaciones hacia la docencia en secundaria en la etapa formativa inicial (Pontes, Ariza y Rey, 2010), que corresponde con el Máster de Formación del Profesorado de Enseñanza Secundaria (MFPES) para España. De este modo, el objetivo de este trabajo es conocer qué tipología de estudiante accede a este máster a partir de su perfil motivacional, basándonos en su grado de autopercepción como futuro docente. A partir de la teoría de la autodeterminación (Ryan y Deci, 2000), que sugiere la existencia de distintos perfiles motivacionales en los individuos, tratamos de identificar qué factores motivacionales se encuentran asociados a los posibles futuros docentes en la enseñanza secundaria, así como identificar tipologías de candidatos según sus características sociodemográficas. Se ha utilizado como fuente de datos, un trabajo de campo consistente en una 
encuesta realizada a los alumnos del MFPES de tres universidades españolas a lo largo del curso 2015-2016.

Con los objetivos descritos anteriormente, el resto del trabajo se articula de la siguiente manera: en segundo apartado se presenta la revisión de la literatura y se desarrollan las preguntas de investigación; en el tercero se describe el método de investigación seguido; y finalmente se exponen los principales resultados del estudio y la discusión de los mismos, de acuerdo con la teoría planteada.

\section{REVISIÓN DE LA LITERATURA}

La calidad educativa se ha convertido en eje central de las políticas públicas en los países más desarrollados como vector de integración económica y social (Cochran-Smith, 2009; Hanushek y Woessmann, 2010). En las últimas décadas, se está produciendo un cambio dentro del modelo educativo español hacia una mayor armonización con los países de su entorno. Esto ha supuesto que la educación se haya convertido en el objetivo prioritario de las políticas educativas nacionales, siendo la formación, incorporación y retención del talento indispensables en las estrategias de reforma: "necesitamos que nuestros sistemas educativos sean capaces de atraer a los mejores candidatos para convertirse en docentes» (Marcelo, 2009, p. 66).

La formación y preparación del profesorado han sido estudiadas desde diversas vertientes como son, por ejemplo: el enfoque de la formación y productividad del alumnado (Harris y Sass, 2011; Sarramona, 2007); las competencias docentes (Perrenoud, 2001; Tribó, 2008); la cualificación del profesorado que imparte la docencia en las especialidades didácticas del máster de formación del profesorado (Martín-Vegas, 2015); el análisis de las políticas de formación (Donnelly y Watkins, 2011; Fernández-Berrueco y Sánchez-Tarazaga, 2014); los modelos comparativos de la formación inicial del profesorado (Lorenzo-Vicente et al, 2015); desde la perspectiva de la evolución histórica de la formación del profesorado (González-Bertolín y Sanz-Ponce, 2014); o incluso, la formación docente en el Marco Europeo de Educación Superior (Bausela, 2010).

En su conjunto, se puede considerar la existencia de un consenso sobre la importancia del papel de la enseñanza inicial del profesorado y su relación con la calidad de la enseñanza y el aprendizaje en los alumnos (Aaronson, Barrow y Sanders, 2007; Darling-Hammond, 2012; Marcelo, 2010; Sahlberg, 2014; Valcarcel, 2005; entre otros). 


\section{Formación inicial}

Aunque desde el punto de vista del contexto político-legislativo, el proceso formativo del profesorado se encuentra intrínsecamente «condicionado por las influencias tanto de la política educativa como por lo que se considera un profesor competente según el desarrollo profesional» (Cano, Orejudo y Cortés, 2012, p. 122). Se debe contar con una buena formación inicial que les asegure las competencias precisas para la docencia, ya que toda sociedad necesita buenos profesores que cumplan los estándares profesionales de calidad (Marcelo, 2010). Pero de acuerdo con García-Garrido (1999), las actitudes y aptitudes no se encuentran presentes de igual forma en todas las personas, de modo que no se puede llegar a considerar que están en disposición para ejercer la función docente con igual resultado.

El conocimiento profesional que debe tener el profesorado es determinante para la efectividad de la enseñanza (Mahler, Großschedl y Harms, 2017). Sin embargo, aunque la formación científica era suficiente tiempo atrás, la complejidad y polivalencia de la labor docente convierte en necesaria la formación didáctica. En este sentido, Bolívar y Moreno (2006) consideran que la formación como especialista disciplinar y la posterior dedicación a la docencia, no permiten la configuración identitaria del profesorado. Es por ello por lo que el docente debe ser algo más que «profesor de una asignatura», asumiendo el rol de educador (Bolívar, 2007), lo que se consigue únicamente por medio de una buena formación, seguida de una motivación específica hacia la enseñanza. De este modo, la formación inicial del profesorado se convierte en un proceso formativo necesario, para dotar a los estudiantes de estas competencias didácticas y pedagógicas básicas para impartir docencia (Molina y Esteve, 2016; Serrano y Pontes, 2017).

Como consecuencia de los problemas subyacentes en la formación inicial del profesorado de educación secundaria, surge la necesidad de hacer un diagnóstico, para poder ofrecer soluciones que ayuden a recalcar el importante papel que tiene este eslabón del proceso formativo, en la vertebración de una educación de calidad. La implantación y desarrollo del MFPES en los últimos años, permite, a pesar del corto periodo transcurrido, explorar su situación actual y tratar de evaluar el grado de cumplimiento de sus objetivos iniciales e identificar los retos que tiene pendientes de resolver en el futuro.

\section{Motivaciones del profesorado}

La literatura científica se ha referido frecuentemente a objetivos altruistas, a la vocación de servicio y otras motivaciones intrínsecas como 
razones principales por las que los estudiantes eligen la enseñanza como carrera profesional (Biscarri, Filella y Jové, 2006; Brookhart y Freeman, 1992). Donde la motivación hace referencia a conceptos como la «energía, dirección, persistencia y equifinalidad», aspectos todos ellos relacionados con «la activación y la intención» del individuo a la consecución de un determinado objetivo por medio de su acción (Ryan y Deci, 2000, p. 69).

Es decir, la motivación intrínseca del futuro docente tiene sus raíces en la autodeterminación u orientación motivacional para la profesión docente. Según Neves de Jesus y Lens (2005), el profesor intrínsecamente motivado emprende y completa tareas profesionales por su valor inherente, como un fin en sí mismo. Por lo tanto, la motivación intrínseca del profesor influye en el valor asignado a los objetivos perseguidos en el proceso de enseñanza/ aprendizaje.

En este sentido, la presencia de una motivación intrínseca o vocacional en el profesorado posee un efecto positivo en la calidad del sistema educativo y, en particular, en el rendimiento del alumnado. Sin embargo, su ausencia no tiene por qué ser la causa de fracaso, ya que existen otros factores que pueden influir, como son la insatisfacción o el absentismo escolar (Larrosa, 2010). Además, la vocación docente no se puede considerar como estática, pues no se nace con ella, se puede adquirir o perder, cuando se idealiza desmesuradamente la docencia (Larrosa, 2010).

La literatura en el área (König y Rothland, 2012; Kyriacou y Coulthard, 2000; Sinclair, 2008, entre otros), señala que las motivaciones principales para elegir la enseñanza como profesión son tres (1) motivaciones altruistas: estar al servicio de las personas, la sociedad o tratar de ver la enseñanza como un trabajo socialmente valioso e importante; (2) motivaciones intrínsecas: abarcan aspectos de la actividad laboral en sí, satisfacción personal, y deseo y amor a la profesión; y (3) motivaciones extrínsecas: cubren aspectos del trabajo que no son inherentes a la profesión docente en sí, como son la garantía de empleo, vacaciones y el salario. Pero se observa que existen diferencias en las motivaciones que dominan en el acceso al profesorado entre los países más desarrollados y menos desarrollados. Si en los primeros, preferentemente, influyen las motivaciones intrínsecas y altruistas, en los menos desarrollados las motivaciones extrínsecas son muy importantes (Bastick, 2000; OCDE, 2005). Sin olvidar que las motivaciones profesionales pueden ser intrínsecas y extrínsecas a la vez (Biscarri, 1993; Özbek, 2007).

De la misma forma, las imágenes negativas del trabajo de los profesores en los medios de comunicación de masas, la ideología política y la opinión 
pública influyen también en la popularidad y la reputación de la enseñanza como opción de carrera profesional (Watt y Richardson, 2008).

\section{MÉTODO DE INVESTIGACIÓN}

En relación con esta problemática, y en el contexto de la formación inicial del futuro profesorado en enseñanza secundaria en España, que se realiza con el MFPES, se ha realizado este estudio mediante la medición de las autopercepciones de los estudiantes de este máster, como posibles docentes y en base a sus características sociodemográficas, con el objeto de contestar a las siguientes preguntas de investigación: ¿se puede confirmar la existencia de diferencias motivacionales significativas en la decisión de querer acceder a la docencia en enseñanza secundaria por el alumnado del máster?, ¿es posible caracterizar socio-demográficamente los candidatos hacia la docencia desde esta perspectiva motivacional? Para dar respuesta a estas preguntas, se expone seguidamente el proceso para el diseño de la herramienta de investigación y recogida de información.

\section{Muestra y recogida de datos}

La fuente de información primaria empleada ha consistido en la recogida de datos por medio de un cuestionario dirigido a potenciales docentes que se encontraban en su fase de formación inicial. Concretamente la encuesta se dirigió a los alumnos del MFPES durante los meses de noviembre de 2015 a enero de 2016 en las Universidades de Burgos (UBU), Córdoba (UCO) y Jaén (UJA), la primera situada en el norte de España y las dos últimas en el sur, dado que, en un estudio de este tipo, la "posibilidad de captar la heterogeneidad es el principal valor añadido a la investigación de la cuestión en general» (Carabaña y de la Fuente, 2016, p. 987). Dichas universidades fueron seleccionadas, para formar parte de este estudio por su amplia tradición en la implantación del MFPES desde el curso 2009/10.

Los contenidos del cuestionario se basan en investigaciones previas (Córdoba, Ortega y Pontes, 2009; Pontes, Serrano y Poyato, 2013; Puentes, Botia y Verdejo, 2015; Watt y Richardson, 2008), aunque se ha adaptado con el objeto de poder recabar información sobre la motivación y expectativas sobre la carrera docente. Este cuestionario se dividió en cuatro bloques: (1) análisis de las características sociodemográficas del alumnado encuestado; (2) motivación; (3) perspectivas; (4) expectativas acerca de la profesión. Se utilizaron preguntas cerradas para elegir entre distintas opciones, preferentemente en el estudio socioeconómico de los alumnos y cuestiones de valoración, utilizando una escala Likert de 1 a 5 puntos para 
evaluar las motivaciones, perspectivas y expectativas de la profesión. Para la selección de los encuestados se utilizó la metodología del muestreo aleatorio simple entre el alumnado presente.

La programación temporal de los cuestionarios tuvo en cuenta que el hecho de la recogida de información coincidiera con la práctica finalización de la formación psicopedagógica genérica en cada una de las universidades, bajo la presunción de que habría pasado tiempo suficiente para que los estudiantes se hubieran formado un criterio informado sobre la formación que estaban recibiendo. El cuestionario fue cumplimentado por el propio alumnado de manera presencial durante las sesiones del máster, con la presencia de los investigadores para resolver las dudas que pudieron ser planteadas por los encuestados al rellenarla.

Tabla 1

Ficha técnica de estudio

\begin{tabular}{|c|c|}
\hline Proceso metodológico & $\begin{array}{c}\text { Encuestas a través de cuestionarios estructurados } \\
\text { y auto-administrados }\end{array}$ \\
\hline Tipo de preguntas & $\begin{array}{l}\text { Cerradas y semi-abiertas de tipo múltiples y de respuesta } \\
\text { única. }\end{array}$ \\
\hline $\begin{array}{c}\text { Tiempo de } \\
\text { cumplimentación } \\
\text { del cuestionario }\end{array}$ & 15 minutos. \\
\hline Universo población & 690 personas (UBU, UCO y UJA). \\
\hline Muestra objetivo & 530 encuestas. \\
\hline Muestra real & 512 encuestas. \\
\hline Forma de contacto & $\begin{array}{l}\text { Se han cumplimentado en el aula con los responsables del } \\
\text { estudio. }\end{array}$ \\
\hline Método de muestreo & Muestreo aleatorio simple por universidad participante. \\
\hline Precisión mínima & $\pm 2,5 \%$ en alumnos/as UBU, UCO y UJA. \\
\hline Nivel de confianza & $95 \%$ \\
\hline $\begin{array}{l}\text { Fecha de prueba } \\
\text { piloto }\end{array}$ & $\begin{array}{l}\text { Realizada en octubre de } 2015 \text { con alumnado de la Univer- } \\
\text { sidad de Burgos. Total: } 10 \text { personas. }\end{array}$ \\
\hline $\begin{array}{l}\text { Fecha de trabajo } \\
\text { de campo }\end{array}$ & Desde noviembre de 2015 a marzo de 2016. \\
\hline $\begin{array}{l}\text { Captura de la } \\
\text { información }\end{array}$ & Se ha realizado por los responsables del estudio. \\
\hline $\begin{array}{l}\text { Tratamiento de la } \\
\text { información }\end{array}$ & $\begin{array}{l}\text { Informático a través del paquete estadístico SPSS } \\
\text { (versión 22.0) }\end{array}$ \\
\hline
\end{tabular}


Previamente se efectuó un pre-test, con el fin de asegurar la comprensión y calidad del cuestionario. La tasa de rechazos a ser encuestados fue baja y no significativa en función de ninguna variable. El tamaño muestral fue de 512 cuestionarios, de las cuales se validaron 503, lo cual supone una muestra suficiente para un adecuado funcionamiento de la técnica estadística de análisis factorial exploratorio (Hair, Anderson, Tatham y Black, 2004). No se ha realizado estratificación por ninguna variable (sexo, edad, formación, etc.). El error muestral, para un nivel de confianza del $95 \%$, es de $\pm 2,5 \%$. Los datos recogidos fueron organizados, tabulados y analizados usando el programa SPSS 22.0. En la tabla 1 se presenta la ficha técnica de la investigación.

En el análisis de datos, las preguntas cerradas se tabularon a partir de las puntuaciones directas otorgadas por los participantes en el estudio. Una vez que los datos fueron tabulados, se aplicaron los diferentes análisis estadísticos.

\section{RESULTADOS}

Los datos demográficos de la muestra reflejan un alumnado formado mayoritariamente por mujeres (62\%), así como por graduados de 25 años en adelante $(55,4 \%)$, siendo la media de edad en torno a los 26 años, congruente con estudios previos en el contexto español (Pontes, et al., 2010). El 40,7\% son egresados en el ámbito de las Artes y Humanidades, frente a un $17,2 \%$ con titulaciones de Ciencias. Es destacable el importante porcentaje de alumnado con estudios en el ámbito técnico, es decir, Ingenierías y Arquitectura (18,5\%). Además, el 20,2\% afirmó que ya tenía previamente otro posgrado y el 30,9\% compaginaba su formación de máster con un trabajo o una beca de inserción laboral.

\section{Análisis factorial confirmatorio}

En términos generales, los datos, resultados y conclusiones que se presentan en este trabajo se refieren al análisis del cuestionario que hace referencia a las motivaciones a la hora de elegir la docencia como futuro profesional. Una vez tabulada la información, se determinó la validez de las respuestas mediante un análisis de confiabilidad y consistencia interna de las variables del cuestionario, a través del coeficiente Alfa de Cronbach, dando como resultado un valor superior a 0,7 , lo que indica una consistencia interna suficiente entre los elementos de la escala. Posteriormente, se recurrió a la técnica estadística multivariante de análisis factorial de componentes principales y una rotación de tipo Varimax. Esta es la metodología más 
común en este tipo de investigaciones (López-González, Pérez-Carbonell y Ramos-Santana, 2011; Hair et al, 2004) que tiene el objeto de reducir las variables objeto de estudio.

Tabla 2

Matriz de Componentes Rotados

\begin{tabular}{|c|c|c|c|}
\hline & $\begin{array}{c}\text { Factor } \\
1 \\
\end{array}$ & $\begin{array}{c}\text { Factor } \\
2 \\
\end{array}$ & $\begin{array}{c}\text { Factor } \\
3 \\
\end{array}$ \\
\hline $\begin{array}{l}\text { (M1) La docencia me interesa porque no tengo otras } \\
\text { salidas profesionales }\end{array}$ & 0,758 & & \\
\hline $\begin{array}{l}\text { (M2) Aunque inicialmente aspiraba a otra profesión, } \\
\text { ahora la docencia me parece muy interesante }\end{array}$ & 0,659 & & \\
\hline $\begin{array}{l}\text { (M3) La docencia en Educ. Secundaria interesa princi- } \\
\text { palmente por sus condiciones de trabajo: estabilidad, } \\
\text { vacaciones,.. }\end{array}$ & 0,692 & & \\
\hline $\begin{array}{l}\text { (M4) En mi entorno no existe una buena opinión sobre } \\
\text { la enseñanza }\end{array}$ & & 0,745 & \\
\hline $\begin{array}{l}\text { (M5) El docente de Educación Secundaria tiene pocos } \\
\text { estímulos profesionales }\end{array}$ & & 0,777 & \\
\hline $\begin{array}{l}\text { (M6) Tengo mucho interés profesional en ejercer la do- } \\
\text { cencia en Secundaria }\end{array}$ & & & 0,319 \\
\hline (M7) La docencia motiva porque se trabaja con jóvenes & & & 0,821 \\
\hline Autovalores & 2,074 & 1,315 & 1,000 \\
\hline$\%$ Varianza & 29,629 & 18,786 & 14,291 \\
\hline$\%$ Varianza acumulada & 29,629 & 48,415 & 62,706 \\
\hline
\end{tabular}

Adecuación muestral Kaiser-Meyer-Olkin (KMO): 0,636

Prueba de esfericidad de Bartlett: $\chi^{2}=575,676$; gl: 45; Sig.: 0,000

En la tabla 2, se presentan los resultados del análisis factorial sobre los ítems de motivaciones. El valor de Kaiser-Meyer-Olkin (KMO), que tiene en cuenta las correlaciones entre variables, es de 0,636 y está por encima del mínimo recomendable. Con este análisis factorial, los 7 ítems iniciales quedaron reducidos a tres factores. Estos resultados permiten intuir la existencia de tres tipos de factores motivacionales que animan o desaniman la elección de los estudios iniciales de formación del profesorado en enseñanza secundaria. El primer factor de la matriz de componentes rotados agrupa a las motivaciones relacionadas con las condiciones de trabajo que se esperan en una futura docencia, o a la consideración de la 
docencia como una posible salida laboral más. Se ha denominado «factores extrínsecos positivos», y explica el 29,63\% de la varianza total. El segundo factor observado hace referencia a aspectos negativos relacionados con la opinión que, tanto el encuestado como los familiares o amigos, tienen sobre la imagen del docente en la sociedad y/o sus escasos estímulos profesionales. Este factor explica el $18,79 \%$ de la varianza total y se ha llamado «factores extrínsecos negativos». El tercer factor de la matriz de componentes rotados, con una varianza explicada del 14,29\% de la varianza total, agrupa los motivos intrínsecos positivos docentes y las motivaciones altruistas. A este tercer factor se le ha denominado «factores intrínsecos».

\section{Análisis clúster}

Posteriormente, los factores motivacionales observados en el análisis factorial se tomaron como base para tratar de determinar la posible existencia de alguna tendencia de agrupación, con el objetivo de comprobar si existen perfiles socio-demográficos definidos en el alumnado y permitir identificar sus rasgos definitorios. Para ello se utilizó un análisis clúster, con el fin de agrupar a los encuestados según las motivaciones expresadas en sus respuestas.

Se recurrió a la prueba MANOVA para comprobar la significatividad de los efectos y la pertenencia al clúster de las variables utilizadas. Las diferencias entre pares de valores se identificaron mediante la utilización del test post hoc de Tukey. Posteriormente, el test Chi-cuadrado se empleó para medir las características sociodemográficas medidas de los estudiantes encuestados (p.e. género, edad, universidad, compaginación de estudios con trabajo, otros estudios de posgrado). Por otra parte, mediante el test ANOVA se realizó una comprobación de las variables. En la tabla 3 se presentan los resultados alcanzados. 
Tabla 3

Resultados del análisis clúster

\begin{tabular}{|c|c|c|c|c|c|c|c|c|}
\hline \multirow{2}{*}{ Componente } & \multirow{2}{*}{$\begin{array}{l}\text { Variables } \\
\text { motiva- } \\
\text { cionales }\end{array}$} & \multicolumn{3}{|c|}{ Clúster de pertenencia } & \multicolumn{2}{|c|}{ Anova } & \multicolumn{2}{|c|}{$\begin{array}{c}\text { Estadístico } \\
\text { igualdad de } \\
\text { varianzas }\end{array}$} \\
\hline & & $\begin{array}{c}1 \\
n=123\end{array}$ & $\begin{array}{c}2 \\
n=168\end{array}$ & $\begin{array}{c}3 \\
n=209\end{array}$ & $\mathbf{F}$ & Sig. & Levene & Sig. \\
\hline \multirow{3}{*}{$\begin{array}{c}\text { Factores } \\
\text { extrínsecos } \\
\text { positivos }\end{array}$} & M1 & $2,89^{(*)}$ & $2,50^{(*)}$ & $1,41^{(*)}$ & 95,582 & $<, 001$ & 54,46 & $<, 001$ \\
\hline & M2 & $3,46^{(*)}$ & $4,13^{(*)}$ & $1,50^{(*)}$ & 541,326 & $<, 001$ & 11,30 & $<, 001$ \\
\hline & M3 & $3,55^{(*)}$ & $3,04^{(*)}$ & $2,10^{(*)}$ & 86,715 & $<, 001$ & 1,05 & ,353 \\
\hline \multirow{2}{*}{$\begin{array}{c}\text { Factores } \\
\text { extrínsecos } \\
\text { negativos }\end{array}$} & M4 & $3,21^{(*)}$ & $1,39^{(*)}$ & $1,94^{(*)}$ & 124,347 & $<, 001$ & 22,02 & $<, 001$ \\
\hline & M5 & $3,42^{(*)}$ & $2,23^{(*)}$ & $2,57^{(*)}$ & 47,958 & $<, 001$ & 5,23 & $<, 010$ \\
\hline \multirow{2}{*}{$\begin{array}{c}\text { Factores } \\
\text { intrínsecos }\end{array}$} & M6 & $3,77^{(*)}$ & $4,29^{(*)}$ & $4,68^{(*)}$ & 52,434 & $<, 001$ & 20,60 & $<, 001$ \\
\hline & M7 & $3,08^{(*)}$ & $3,47^{(*)}$ & $3,63^{(*)}$ & 11,522 & $<, 001$ & 0,66 & ,0936 \\
\hline
\end{tabular}

(*) Variable significativa al 0,001.

El análisis clúster determinó la existencia de tres tipologías distintas de estudiantes que pretenden ser profesores de educación secundaria. El primer conglomerado o clúster estaría compuesto por un total de 123 participantes (24,6\% del total), y presenta las mayores puntuaciones en las variables M1 y M3 y niveles elevados para M2, que se corresponde a las variables de motivación extrínseca positiva hacia la docencia, pero también es el grupo que tiene, con diferencia, las mayores puntuaciones en los factores extrínsecos negativos (M4 y M5). A este grupo se le ha denominado alumnado con motivación circunstancial, ya que no tiene buena opinión de la docencia, y lo toma simplemente como una salida profesional viable. El clúster 2 , compuesto por 168 participantes $(33,6 \%)$, presenta la mayor valoración para la motivación M2 «aunque inicialmente aspiraba a otra profesión, ahora la docencia me parece interesante». Asimismo, este grupo se caracteriza por tener altas puntuaciones en los factores intrínsecos relacionados con la docencia, así como muy baja puntuación en los factores extrínsecos negativos, a este grupo se le ha denominado alumnado con vocación encontrada. Finalmente, el clúster 3, formado por 209 participantes $(41,8 \%)$, presenta las puntuaciones más altas en las variables que conforman los factores intrínsecos, tanto para M6 como para M7, así como puntuaciones bajas tanto en los factores extrínsecos positivos como extrínsecos negativos, a este grupo se le ha denominado alumnado con vocación docente intrínseca. 
Posteriormente, se asignó a cada variable sociodemográfica el número de individuos que pertenecían a cada clúster, con el objeto de comprobar si existía un patrón identificable entre el alumnado (tabla 4). Para detectar esta posible relación se realizó un análisis Chi-cuadrado de Pearson.

Tabla 4

Características demográficas por grupo clúster

\begin{tabular}{|c|c|c|c|c|}
\hline Características demográficas & $\begin{array}{c}\text { Clúster } 1 \\
(\mathrm{n}=123) \\
n(\%)\end{array}$ & $\begin{array}{c}\text { Clúster } 2 \\
(\mathrm{n}=168) \\
n(\%)\end{array}$ & $\begin{array}{c}\text { Clúster } 3 \\
(\mathrm{n}=209) \\
n(\%)\end{array}$ & Total \\
\hline \multicolumn{5}{|l|}{ Género } \\
\hline Hombre & $53(27,9 \%)$ & $59(31,1 \%)$ & $78(41,1 \%)$ & 190 \\
\hline Mujer & $70(22,6 \%)$ & $109(35,2 \%)$ & $131(42,3 \%)$ & 310 \\
\hline \multicolumn{5}{|l|}{ Edad } \\
\hline Hasta 24 & $41(18,6 \%)$ & $58(26,2 \%)$ & $122(55,2 \%)$ & 221 \\
\hline $25-29$ & $47(27,5 \%)$ & $70(40,9 \%)$ & $54(31,6 \%)$ & 171 \\
\hline $30-34$ & $19(34,5 \%)$ & $21(38,2 \%)$ & $15(27,3 \%)$ & 55 \\
\hline $35-39$ & $10(32,3 \%)$ & $11(35,5 \%)$ & $10(32,3 \%)$ & 31 \\
\hline$>40$ & $4(22,2 \%)$ & $8(44,4 \%)$ & $6(33,3 \%)$ & 18 \\
\hline \multicolumn{5}{|l|}{ Estudios previos } \\
\hline Artes y humanidades & $32(17,1 \%)$ & $41(21,9 \%)$ & $114(61,0 \%)$ & 187 \\
\hline Ciencias & $24(30,4 \%)$ & $32(40,5 \%)$ & $23(29,1 \%)$ & 79 \\
\hline Ciencias de la Salud & $5(25,0 \%)$ & $11(55,0 \%)$ & $4(20,0 \%)$ & 20 \\
\hline Ciencias Sociales y Jurídicas & $22(25,0 \%)$ & $36(40,9 \%)$ & $30(34,1 \%)$ & 88 \\
\hline Ingeniería y Arquitectura & $27(31,8 \%)$ & $35(41,2 \%)$ & $23(27,1 \%)$ & 85 \\
\hline \multicolumn{5}{|l|}{ Otro posgrado } \\
\hline Sí & $37(37,0 \%)$ & $41(41,0 \%)$ & $22(22,0 \%)$ & 100 \\
\hline No & $81(20,6 \%)$ & $126(32,0 \%)$ & $187(47,5 \%)$ & 394 \\
\hline \multicolumn{5}{|l|}{ Dedicación } \\
\hline Solo estudiar & $70(23,0 \%)$ & $94(30,9 \%)$ & $140(46,1 \%)$ & 304 \\
\hline Estudiar y trabajar & $53(27,3 \%)$ & $72(37,1 \%)$ & $69(35,6 \%)$ & 194 \\
\hline \multicolumn{5}{|l|}{ Universidad } \\
\hline Burgos & $29(31,2 \%)$ & $31(33,3 \%)$ & $33(35,5 \%)$ & 93 \\
\hline Córdoba & $65(21,0 \%)$ & $103(33,2 \%)$ & $142(45,8 \%)$ & 310 \\
\hline Jaén & $29(30,2 \%)$ & $34(35,4 \%)$ & $33(34,4 \%)$ & 96 \\
\hline
\end{tabular}

Notas: (1) la descripción porcentual es en horizontal;

(2) el número de observaciones en Ciencias de la Salud es reducido para emitir un juicio de valor. 
En referencia al género, aunque el alumnado es mayoritariamente femenino (62\%), el sexo de los encuestados no es significativo para su pertenencia a algún clúster $\left(\chi^{2}(2, N=500)=1,99, p=0,371\right)$. Es decir, no se puede afirmar que exista una mayor vocación docente en un género que en el otro. Al realizar el mismo estudio por tramos de edad, sí se observa relación significativa entre la edad y la pertenencia a alguno de los clústeres $\left(\chi^{2}(5, N=496)=31,70, p<0,001\right)$. Concretamente, el alumnado más joven, hasta 24 años, que tiene su formación de grado recién terminada, tiene un mayor perfil de pertenencia al clúster 3 , en la que la vocación docente intrínseca y altruista es mayor, frente a otras cohortes de edad, donde hay mayor proporción de alumnado asignado al clúster 2 . Resulta interesante que, a partir de los 30 años, existe un importante número de alumnos pertenecientes al clúster 1 , donde la motivación para acceder a la docencia es meramente circunstancial.

Las áreas de conocimiento previas con que el alumnado accede al máster de formación de profesorado de enseñanza secundaria, también guardan relación con la asignación a los clústeres de agrupación motivacional $\left(\chi^{2}(5, N=498)=48,52, p<0,001\right)$. Es destacable la elevada proporción de estudiantes de Artes y Humanidades que pertenecen al grupo con mayor motivación intrínseca $(61,0 \%)$; frente al alumnado con formación en áreas como las de Ciencias, Ciencias Sociales y Jurídicas, Ingenierías o Arquitectura donde prima la vocación encontrada, pero con un importante grupo de alumnos con motivación puramente circunstancial. De forma similar, existe relación entre las variables, tener otro posgrado previo al máster de formación de profesorado y la pertenencia a un clúster $\left(\chi^{2}(2, N=498)=35,48, p<0,001\right)$, el alumnado que no tiene otro posgrado suele tener más motivaciones intrínsecas. Por otra parte, el tipo de dedicación (compartida o no) durante el máster $\left(\chi^{2}(2, N=498)=5,35, p<\right.$ $0,1)$ no condiciona la pertenencia a un determinado clúster. Finalmente, el tipo de universidad de pertenencia de los encuestados $\left(\chi^{2}(3, N=499)=7,9\right.$, $p<0,1)$, tampoco condiciona la pertenencia a un determinado clúster, lo que implica que el origen geográfico del alumnado no tiene relación con la motivación.

\section{DISCUSIÓN Y CONCLUSIONES}

En este trabajo se ha intentado identificar, por un lado, las motivaciones que impulsan al alumnado a realizar el MFPES, entendiendo esta formación, como previa a un posible futuro profesional docente en educación secundaria; y por otro, si es posible caracterizar sociodemográficamente las tipologías de los candidatos desde esta perspectiva motivacional. 
En referencia a la primera pregunta de investigación, se ha podido definir la existencia de tres tipos de factores motivaciones en los estudiantes que acceden a esta formación, en las universidades estudiadas: 1) factores motivacionales intrínsecos y altruistas, 2) factores motivaciones extrínsecos, estos dos factores motivacionales ya definidos en los estudios de König y Rothland (2012), pero también se ha detectado la presencia de 3) factores motivacionales extrínsecos negativos sobre la profesión docente (Watt y Richardson, 2008).

En función de las motivaciones expresadas, respecto a la carrera docente de los encuestados, se han caracterizado tres grupos o tipologías de alumnado que accede a este máster: el primer grupo estaría formado por estudiantes que consideran la docencia como una mera salida profesional, es decir, una motivación circunstancial ante el actual mercado laboral, representando casi la cuarta parte de los encuestados. El segundo grupo estaría formado por alumnado que no había considerado inicialmente la docencia como proyecto profesional de futuro, pero una vez en el máster, han encontrado satisfacción en la docencia, es decir, una vocación encontrada. El tercer grupo, el más numeroso, se caracteriza por tener una vocación intrínsecamente docente.

En cuanto a la presencia de la mujer en la docencia, es superior a la de los hombres (Azman, 2013). En consecuencia, también hay mayor presencia femenina en el máster, pero el género de los encuestados no influye en su adscripción a los grupos definidos previamente y, en consecuencia, se intuye que no existe una mayor vocación docente intrínseca según el género. Tampoco hay evidencia de que influya el origen geográfico del encuestado, ni el hecho de realizar otra actividad remunerada además de su formación en el máster.

Los resultados revelan que existe relación significativa entre la edad y el grupo motivacional de pertenencia, siendo el alumnado más joven donde se ha encontrado mayor motivación intrínseca. Además, los estudiantes procedentes de titulaciones relacionadas con las Artes y Humanidades presentan mucha mayor motivación intrínseca que el resto de sus compañeros, especialmente respecto a los alumnos procedentes de Ciencias e Ingeniería. Por otra parte, los datos indican que tener previamente otros estudios de posgrado, tiene relación con el hecho de pertenecer a los grupos con menor motivación intrínseca respecto a la docencia, debido quizás, a que se considera como una segunda opción ante la falta de salidas en otras profesiones.

El conocimiento de la existencia, por parte de las autoridades educativas, de las diversas motivaciones a la hora de comenzar la carrera docente en la educación secundaria debería tenerse en cuenta para la 
selección, elaboración y planificación de la formación inicial del futuro profesorado, permitiendo espacios de desarrollo y fomento de su dimensión vocacional. En este sentido resulta necesario, como apuntan Balan, Bujor y Serdenciuc (2012), que se trabaje sobre la base de las motivaciones, actitudes y expectativas de los candidatos a profesores (p.e. aumentar el periodo de prácticas en centros), para que adquieran las competencias necesarias para la formación del futuro y poder así trabajar en línea con los principios de competitividad y calidad por los que se rige el Espacio Europeo de Educación Superior. Y quizás, se deba plantear la conveniencia de establecer para algunos estudios de grados, itinerarios formativos especialmente orientados a la enseñanza secundaria.

Como limitaciones principales de este estudio entendemos que, aunque se ha identificado los diferentes perfiles motivacionales del alumnado del MFPES y sus características sociodemográficas asociadas, mediante una investigación transversal, tal vez estos resultados podrían ser mejor explicados a través de estudios longitudinales, que permitieran tener una visión más detallada sobre el proceso de formación del profesorado de educación secundaria, tanto a través de estudios de tipo cuantitativo como cualitativo. Finalmente, se debe destacar como limitación principal, el número de universidades consultadas (tres), por lo que el grado de generalización geográfica del estudio a todo el ámbito geográfico español es relativo. 


\section{REFERENCIAS BIBLIOGRÁFICAS}

Aaronson, D., Barrow, L. \& Sander, W. (2007). Teachers and student achievement in the Chicago public high schools. Journal of Labor Economics, 25(1), 95-135.

Azman, N. (2013). Choosing teaching as a career: Perspectives of male and female Malaysian student teachers in training. European Journal of Teacher Education, 36(1), 113-130.

Balan, C.C., Bujor, L. \& Serdenciuc, N.L. (2012). The role of the initial psychopedagogical training in structuring motivational and attitudinal dynamic toward teaching career. ProcediaSocial and Behavioral Sciences, 33, 463-467.

Bastick, T. (2000). Why teacher trainees choose the teaching profession: Comparing trainees in metropolitan and developing countries. International Review of Education, 46(3/4), 343-349.

Báusela, E. (2010). Planes de acción tutorial en la universidad. Revista de la educación superior, 39 (153), 119-122.

Biscarri, J. (1993). Motivaciones de los profesores respecto a su formación permanente. Revista interuniversitaria de formación del profesorado, 18, 221237.

Biscarri, J., Filella, G. y Jové, G. (2006). Factores relacionados con la percepción de la calidad docente del profesorado universitario. Revista Interuniversitaria de Formación del Profesorado, 20(3), 287-309.

Bolívar, A. \& Moreno, J.M. (2006). Between transaction and transformation: The role of school principals as education leaders in Spain. Journal of Educational Change, 7(1-2), 19-31.
Bolívar, A. (2007). La formación inicial del profesorado de secundaria y su identidad profesional. Estudios Sobre Educación, 12, 13-30.

Boyd, D.J., Grossman, P.L., Lankford, H., Loeb, S. \& Wyckoff, J. (2009). Teacher preparation and student achievement. Educational Evaluation and Policy Analysis, 31(4), 416-440.

Brookhart, S.M. \& Freeman, D.J. (1992). Characteristics of entering teacher candidates. Review of Educational Research, 62(1), 37-60.

Caballero, D. \& Huertas, F.J. (2016). Main Motivations of Students of Childhood Education and Primary Education to Become Teachers. Mediterranean Journal of Social Sciences, 7(6), 153-158.

Cano, J., Orejudo, S. y Cortés, A. (2012). La formación inicial del profesorado de Secundaria: primera investigación en el desarrollo del prácticum de la Universidad de Zaragoza. Revista electrónica interuniversitaria de formación del profesorado, 15(3), 121-132.

Carabaña, J. y de la Fuente, G. (2016). Facultad por Facultad. Origen familiar y empleo de los licenciados en CCSS y Humanidades de la UCM en el año 2003. Revista Complutense de Educación, 27(3), 983-1001.

Cochran-Smith, M. (2009). The new teacher education in the United States: directions forward. Teachers and teaching, 14 (4), 271-282.

Comisión Europea (2013). Key Data on Teachers and School Leaders in Europe. 2013 Edition. Eurydice Report. Luxembourg: Publications Office of the European Union. Recuperado de https://goo.gl/21F7tK 
Córdoba, F., Ortega, R. y Pontes, A. (2009). Los universitarios de ciencias ante la docencia en Secundaria como experiencia profesional. Revista de Educación, 348, 401-421.

Darling-Hammond, L. (2012). Powerful teacher education: Lessons from exemplary programs. San Francisco: John Wiley y Sons.

Donnelly, V. \& Watkins, A. (2011). Teacher education for inclusion in Europe. Prospects, 41(3), 341-353.

Esteve, J.M. (2006). La profesión docente en Europa: perfil, tendencias y problemática: La formación inicial. Revista de educación, 340, 19-40.

Fernández-Berrueco, R. y SánchezTarazaga, L. (2014). Competencias docentes en Secundaria. Análisis de perfiles de profesorado. Relieve, 20(1), 1-20.

García-Garrido, J.L. (1999). El profesor del siglo XXI. Bordón. Revista de Orientación Pedagógica, 51(4), 435-447.

González-Bertolín, A. y Sanz-Ponce, R. (2014). De la relevancia de las reformas educativas en la evolución de la formación del profesorado de educación secundaria. Profesorado, Revista de Currículum y Formación del Profesorado, 18(1), 367-381.

Hair, J.F., Anderson, R.E., Tatham, R.L. y Black, W.C. (2004). Análisis Multivariante. Madrid: Pearson.

Hanushek, E.A. \& Woessmann, L. (2010). The economics of international differences in educational achievement. CESifo (Working Paper, N. ${ }^{\circ}$ 3037). Recuperado de: https://www.econstor. $\mathrm{eu} /$.

Harris, D.N. \& Sass, T.R. (2011). Teacher training, teacher quality and student achievement. Journal of public economics, 95(7-8), 798-812.
Hobson, A.J., Ashby, P., Malderez, A. \& Tomlinson, P.D. (2009). Mentoring beginning teachers: What we know and what we don't. Teaching and teacher education: An International Journal of Research and Studies, 25(1), 207-216.

König, J. \& Rothland, M. (2012). Motivations for choosing teaching as a career: Effects on general pedagogical knowledge during initial teacher education. Asia-Pacific Journal of Teacher Education, 40(3), 289-315.

Kyriacou, C. \& Coulthard, M. (2000). Undergraduates' views of teaching as a career choice. Journal of Education for Teaching, 26(2), 117-126.

Larrosa, F. (2010). Vocación docente versus profesión docente en las organizaciones educativas. Revista electrónica interuniversitaria de formación del profesorado, 13(4), 4351.

León, J., Domínguez, E., Núñez, J.L., Pérez, A. y Martín-Albo, J. (2011). Traducción y validación de la versión española de la Echelle de Satisfaction des Besoins Psychologiques en el contexto educativo. Anales de psicología, 27(2), 405-411.

López-González, E., Pérez-Carbonell, A. y Ramos-Santana, G. (2011). Modelos complementarios al Análisis Factorial en la construcción de escalas ordinales: un ejemplo aplicado a la medida del Clima Social Aula. Revista de Educación, 354, 369-397.

Lorenzo-Vicente, J.A., Muñoz-Galiano, I.M. y Beas-Miranda, M. (2015). Modelos de formación inicial del profesorado de Educación Secundaria en España desde una perspectiva europea. Revista Complutense de Educación, 26(3), 741-757. 
Mahler, D., Großschedl, J. \& Harms, U. (2017). Using doubly latent multilevel analysis to elucidate relationships between science teachers' professional knowledge and students' performance. International Journal of Science Education, 39(2), 213-237.

Marcelo, C. (2009). Los comienzos en la docencia: un profesorado con buenos principios. Profesorado. Revista de currículum y formación de profesorado, 13(1), 1-25.

Marcelo, C. (2010). La identidad docente: constantes y desafíos. Revista Interamericana de Investigación, Educación y Pedagogía, RIIEP, 3(1), 15-42.

Martín-Vegas, R.A. (2015). La Didáctica de la Lengua y la Literatura Españolas en el Máster en Formación del Profesorado de Educación Secundaria. Revisión y renovación. Revista española de pedagogía, 73 261), 365379.

Ministerio de Educación, Cultura y Deporte. Gobierno de España (2014). TALIS: 2013. Estudio Internacional de la Enseñanza y el Aprendizaje. Informe Español. Recuperado de: http://www. mecd.gob.es/.

Molina, M.Á. y Esteve, R.P. (2016). El pensamiento del alumnado respecto de la formación inicial en el Máster de Educación Secundaria. XIV Jornadas de Redes de Investigación en Docencia Universitaria. Instituto de Ciencias de la Educación (ICE), Valencia.

Musset, P. (2010). Initial teacher education and continuing training policies in a comparative perspective. OECD Education Working Papers, 48, 1-50.

Neves de Jesus, S. y Lens, W. (2005). An Integrated Model for the Study of Teacher Motivation. Applied Psychology, 54(1), 119-134.

Organización para el Desarrollo Económico y la Cooperación. (2005). Teachers matters: Attracting, developing and retaining effective teachers. Recuperado de http://www.oecd.org/ edu.

Özbek, R. (2007). Teacher candidates' perceptions about importance of personal, economical and social factors which affect their decisions to be teachers. Firat Üniversitesi Sosyal Bilimler Dergisi, 17(1), 145-160.

Perrenoud, P. (2001). La formación de los docentes en el siglo xxI. Revista de Tecnología educativa, 14(3), 503-523.

Pontes, A., Ariza, L. y Rey, R. (2010). Identidad profesional docente en aspirantes a profesorado de enseñanza secundaria. Psychology, Society \& Education, 2(2), 49-60.

Pontes, A., Serrano, R. y Poyato, F.J. (2013). Concepciones y motivaciones sobre el desarrollo profesional docente en la formación inicial del profesorado de educación secundaria. Revista Eureka sobre Enseñanza y Divulgación de las Ciencias, 10, 533-551.

Puentes, R.V., Botia, A.B. \& Verdejo, A.M. (2015). A review of the initial teachers's education in Spain: The masters in secondary education. Educação em Revista, 31(3), 251-278.

Ryan, R.M. \& Deci, E.L. (2000). Selfdetermination theory and the facilitation of intrinsic motivation, social development, and well-being. American psychologist, 55(1), 68-78.

Sahlberg, P. (2014). Facts, true facts and research in improving education systems. Inaugural Annual Lecture. British Educational Research Association (BERA). 
Sarramona, J. (2007). Las competencias profesionales del profesorado de secundaria, Estudios sobre educación, $12,31-40$.

Serrano, R. y Pontes, A. (2017). Diferencias entre expectativas y logros en las competencias del Prácticum del Máster de Formación del Profesorado de Enseñanza Secundaria. Revista Electrónica Interuniversitaria de Formación del Profesorado, 20(1), 1-18.

Sinclair, C. (2008). Initial and changing student teacher motivation and commitment to teaching. Asia-Pacific Journal of Teacher Education, 36(2), 79-104.
Tribó, G. (2008). El nuevo perfil profesional de los profesores de Secundaria, Educación XX1, 11, 183209.

Valcarcel, M. (2005). La preparación del profesorado universitario para la Convergencia Europea en Educación Superior. Educatio Siglo XXI, 23, 209213.

Watt, H.M.G. \& Richardson, P.W. (2008). Motivations, perceptions, and aspirations concerning teaching as a career for different types of beginning teachers. Learning and Instruction, 18(5), 408-428. 


\title{
PERFIL ACADÉMICO Y PROFESIONAL DE LOS AUTORES
}

Guzmán Antonio Muñoz-Fernández. Profesor de Enseñanza Secundaria desde 1991. En el año 2008 se incorporó al Área de Organización de Empresas de la Universidad de Córdoba en comisión de servicios. Ha participado en numerosos grupos de trabajo de innovación educativa en Secundaria y Formación Superior. Colabora con los másteres de Formación del Profesorado y de Comercio Exterior. Sus líneas de investigación principales están relacionadas con turismo cultural, emprendimiento y satisfacción laboral.

Pablo Rodríguez-Gutiérrez. Profesor Ayudante Doctor en el Departamento de Estadística, Econometría, Investigación Operativa, Organización de Empresas y Economía Aplicada de la Universidad de Córdoba desde 2015. Es profesor del área de Organización de Empresas. Su línea de investigación principal está relacionada con la sostenibilidad y el cambio organizacional, aunque también está interesado en temas docentes como son las motivaciones del alumnado del Máster de Formación del Profesorado de EE.SS.

Mercedes Luque-Vílchez. Investigadora Postdoctoral en Departamento de Economía y Administración de Empresas de la Universidad de Burgos desde junio de 2018. Tiene experiencia docente en el área de Economía Financiera y Contabilidad. Su línea de investigación principal está relacionada con la contabilidad social y medioambiental, aunque también está interesada en la investigación de temas puramente docentes como son la formación inicial del profesorado novel.

\author{
Dirección de los autores: Guzmán Antonio Muñoz Fernández \\ Pablo Rodríguez Gutiérrez \\ Facultad de Derecho y CC. Económicas y \\ Empresariales \\ Área de Organización de Empresas \\ C/ Puerta Nueva s/n \\ 14071 - Córdoba \\ E-mail: guzman.munoz@uco.es \\ pablo.rodriguez@uco.es \\ Mercedes Luque-Vílchez \\ Universidad de Burgos \\ Facultad de Ciencias Económicas y \\ Empresariales, Área de Contabilidad \\ Plaza Infanta Elena \\ 09001 - Burgos \\ E-mail: mlvilches@ubu.es
}


92 GUZMÁN ANTONIO MUÑOZ-FERNÁNDEZ, PABLO RODRÍGUEZ-GUTIÉRREZ, MERCEDES LUQUE-VÍLCHEZ LA FORMACIÓN INICIAL DEL PROFESORADO DE EDUCACIÓN SECUNDARIA EN ESPAÑA..

Fecha Recepción del Artículo: 14. Octubre. 2017 Fecha Modificación del Artículo: 18. Marzo. 2018

Fecha Aceptación del Artículo: 22. Marzo. 2018

Fecha revisión para publicación: 20. Junio. 2018 